\title{
High-Order Dispersive Cubic-Quintic Schrödinger Equation and Its Exact Solutions
}

\author{
K. Hosseini ${ }^{a, *}$, R. Ansari ${ }^{b}$, F. SAmadani $^{b}$, A. Zabihi $^{c}$, A. Shafaroody $^{d}$ \\ AND M. MIRZAZADEH ${ }^{e}$ \\ ${ }^{a}$ Department of Mathematics, Rasht Branch, Islamic Azad University, Rasht, Iran \\ ${ }^{b}$ Department of Mechanical Engineering, University of Guilan, P.O. Box 3756, Rasht, Iran \\ ${ }^{c}$ Department of Mechanical Engineering, Ahrar Institute of Technology and Higher Education, Rasht, Iran \\ ${ }^{d}$ Young Researchers and Elite Club, Rasht Branch, Islamic Azad University, Rasht, Iran \\ ${ }^{e}$ Department of Engineering Sciences, Faculty of Technology and Engineering, East of Guilan, University of Guilan, \\ P.C. 44891-63157 Rudsar-Vajargah, Iran \\ (Received January 2, 2019; received version June 10, 2019; in final form July 11, 2019)
}

\begin{abstract}
The light pulses propagation in optical fibers modeled by the dispersive cubic-quintic Schrödinger equation including high-order time derivatives is investigated in detail. For this purpose, the $\exp _{a}$-function scheme is utilized along with a symbolic computation system to gain the exact solutions of the model. As an outcome, a wide range of exact solutions including dark and periodic solitary wave solutions are effectively derived, verifying the excellent performance of the scheme.
\end{abstract}

DOI: 10.12693/APhysPolA.136.203

PACS/topics: dispersive cubic-quintic Schrödinger equation, high-order time derivatives, optical fibers, $\exp _{a^{-}}$ function scheme, dark and periodic solitary wave solutions

\section{Introduction}

The countless applications of nonlinear evolution equations (NLEEs) in diverse scientific fields have attracted the attention of many academic scholars to search and analyze their exact solutions. In this respect, various methods such as the Kudryashov method [1-7], tanh-coth method $[8,9]$, modified simple equation method [10, 11], sine-cosine method [12, 13], transformed rational function method [14, 15], ansatz method [16, 17], auxiliary equation method $[18,19]$, semi-inverse variational method [20, 21], and exp -function method [22-24] have been utilized to solve and handle the NLEEs.

The $\exp _{a}$-function scheme is one of the most practical techniques to acquire the exact solutions of NLEEs and is widely exerted to retrieve the exact solutions of NLEEs; for example, the combined $\mathrm{KdV}-\mathrm{mKdV}$ equation [22], the unstable nonlinear Schrödinger equation [23], and the Tzitzéica-type equations [24].

In the present paper, the dispersive cubic-quintic Schrödinger equation (DCQSE) including higher-order time derivatives is studied through the $\exp _{a}$-function scheme. The nonlinear governing model in its dimensionless form is presented as below [25-27]:

$$
\begin{aligned}
& \mathrm{i} u_{z}-\frac{\alpha_{1}}{2} u_{t t}+\beta_{1}|u|^{2} u= \\
& \quad \mathrm{i} \frac{\alpha_{2}}{6} u_{t t t}+\frac{\alpha_{3}}{24} u_{t t t t}-\beta_{2}|u|^{4} u,
\end{aligned}
$$

\footnotetext{
*corresponding author; e-mail: kamyar_hosseini@yahoo.com
}

which describes the light pulses propagation in optical fibers. In the nonlinear model (1), $z$ is the normalized propagation distance, $t$ is the retarded time, $u(z, t)$ is the slowly varying envelope of the electric field, $\alpha_{1}$ is the second-order dispersion related to the group velocity dispersion, $\alpha_{2}$ is the third-order dispersion, $\alpha_{3}$ is the fourth-order dispersion, and finally $\beta_{1}$ and $\beta_{2}$ are the coefficients of cubic-quintic terms.

Some recent research works related to the model (1) are listed here. Dai et al. [25] employed the generalized projected Riccati expansion method to acquire a wide range of new exact solutions to the model (1). The solitary wave solutions of the model (1) were also gained by Azzouzzi and her colleagues [26] by means of the complex envelope function approach. Xie and his co-workers [27] obtained distinct exact solutions of the model (1) using the complete discrimination system technique.

The organization of this paper is as follows: in Sect. 2, the description of the $\exp _{a}$-function scheme is given. In Sect. 3, the $\exp _{a}$-function scheme is applied to procure the exact solutions of the DCQSE including high-order time derivatives. In Sect. 4, the physical properties of exact solutions are presented. The last section summarizes the results of the current study.

\section{2. $\exp _{a}$ function scheme}

Suppose an NLEE can be presented as

$$
P\left(u, u_{z}, u_{t}, u_{z z}, u_{z t}, u_{t t}, \ldots\right)=0 .
$$

The above Eq. (2) can be converted to the following nonlinear ordinary differential equation:

$$
O\left(\varphi, \varphi^{\prime}, \varphi^{\prime \prime}, \ldots\right)=0
$$


with the assumption

$$
u(z, t)=\varphi(\xi) \mathrm{e}^{\mathrm{i} \theta}, \quad \xi=k z-c t, \quad \theta=\mu z-\omega t .
$$

Let us surmise a non-trivial solution for Eq. (3) in the form below

$$
\varphi(\xi)=\frac{a_{0}+a_{1} a^{\xi}+\cdots+a_{N} a^{N \xi}}{b_{0}+b_{1} a^{\xi}+\cdots+b_{N} a^{N \xi}},
$$

where $a_{i}(0 \leq i \leq N)$ and $b_{i}(0 \leq i \leq N)$ are found later and $N$ is a natural number.

By replacing Eq. (4) in Eq. (3), we get

$$
F\left(a^{\xi}\right)=l_{0}+l_{1} a^{\xi}+\cdots+l_{\tau} a^{\tau \xi}=0 .
$$

Through setting $l_{i}(0 \leq i \leq \tau)$ in $(5)$ to zero, we procure a set of nonlinear equations as follows:

$$
l_{i}=0, \quad i=0, \ldots, \tau .
$$

Ultimately, by solving the yielded system (6), the nontrivial solutions of NLEE (2) are gained.

\section{Nonlinear governing model and its exact solutions}

In this section, by utilizing the following transformation:

$$
u(z, t)=\varphi(\xi) \mathrm{e}^{\mathrm{i} \theta}, \quad \xi=k z-c t, \quad \theta=\mu z-\omega t,
$$

in which $\varphi$ and $\theta$ respectively point out the amplitude component and the phase component of $u$, substituting it into Eq. (1), and recognizing the real and imaginary parts, the model (1) is decomposed into the following higher-order ordinary differential equations:

$$
\begin{aligned}
& 4 c^{3}\left(\alpha_{3} \omega-\alpha_{2}\right) \varphi^{\prime \prime \prime} \\
& +\left(-24 k+24 \alpha_{1} \omega c+12 \alpha_{2} \omega^{2} c-4 \alpha_{3} \omega^{3} c\right) \varphi^{\prime}=0, \\
& \alpha_{3} c^{4} \varphi^{(4)}+\left(12 \alpha_{1} c^{2}+12 \alpha_{2} \omega c^{2}-6 \alpha_{3} \omega^{2} c^{2}\right) \varphi^{\prime \prime} \\
& \quad+\left(24 \mu-12 \alpha_{1} \omega^{2}-4 \alpha_{2} \omega^{3}+\alpha_{3} \omega^{4}\right) \varphi \\
& \quad-24 \beta_{1} \varphi^{3}-24 \beta_{2} \varphi^{5}=0 .
\end{aligned}
$$

\subsection{Scheme and its results}

Through selecting $N=1$, the non-trivial solution (4) becomes

$$
\varphi(\xi)=\frac{a_{0}+a_{1} a^{\xi}}{b_{0}+b_{1} a^{\xi}}, \quad a \neq 1 .
$$

Setting the above non-trivial solution in the higher-order Eqs. (7) and (8) and equating the factor of each power of $a^{\xi}$ in the resulting equations to zero, a nonlinear set is gained as

$$
\begin{aligned}
& (\ln (a))^{2} c^{3} \omega a_{0} \alpha_{3} b_{0}^{2} b_{1}-(\ln (a))^{2} c^{3} \omega a_{1} \alpha_{3} b_{0}^{3}-(\ln (a))^{2} c^{3} a_{0} \alpha_{2} b_{0}^{2} b_{1}+(\ln (a))^{2} c^{3} a_{1} \alpha_{2} b_{0}^{3}-c \omega^{3} a_{0} \alpha_{3} b_{0}^{2} b_{1}+c \omega^{3} a_{1} \alpha_{3} b_{0}^{3} \\
& +3 c \omega^{2} a_{0} \alpha_{2} b_{0}^{2} b_{1}-3 c \omega^{2} a_{1} \alpha_{2} b_{0}^{3}+6 c \omega a_{0} \alpha_{1} b_{0}^{2} b_{1}-6 c \omega a_{1} \alpha_{1} b_{0}^{3}-6 k a_{0} b_{0}^{2} b_{1}+6 k a_{1} b_{0}^{3}=0, \\
& -4(\ln (a))^{2} c^{3} \omega a_{0} \alpha_{3} b_{0} b_{1}^{2}+4(\ln (a))^{2} c^{3} \omega a_{1} \alpha_{3} b_{0}^{2} b_{1}+4(\ln (a))^{2} c^{3} a_{0} \alpha_{2} b_{0} b_{1}^{2}-4(\ln (a))^{2} c^{3} a_{1} \alpha_{2} b_{0}^{2} b_{1}-2 c \omega^{3} a_{0} \alpha_{3} b_{0} b_{1}^{2} \\
& +2 c \omega^{3} a_{1} \alpha_{3} b_{0}^{2} b_{1}+6 c \omega^{2} a_{0} \alpha_{2} b_{0} b_{1}^{2}-6 c \omega^{2} a_{1} \alpha_{2} b_{0}^{2} b_{1}+12 c \omega a_{0} \alpha_{1} b_{0} b_{1}^{2}-12 c \omega a_{1} \alpha_{1} b_{0}^{2} b_{1}-12 k a_{0} b_{0} b_{1}^{2}+12 k a_{1} b_{0}^{2} b_{1}=0 \\
& (\ln (a))^{2} c^{3} \omega a_{0} \alpha_{3} b_{1}^{3}-(\ln (a))^{2} c^{3} \omega a_{1} \alpha_{3} b_{0} b_{1}^{2}-(\ln (a))^{2} c^{3} a_{0} \alpha_{2} b_{1}^{3}+(\ln (a))^{2} c^{3} a_{1} \alpha_{2} b_{0} b_{1}^{2}-c \omega^{3} a_{0} \alpha_{3} b_{1}^{3}+c \omega^{3} a_{1} \alpha_{3} b_{0} b_{1}^{2} \\
& +3 c \omega^{2} a_{0} \alpha_{2} b_{1}^{3}-3 c \omega^{2} a_{1} \alpha_{2} b_{0} b_{1}^{2}+6 c \omega a_{0} \alpha_{1} b_{1}^{3}-6 c \omega a_{1} \alpha_{1} b_{0} b_{1}^{2}-6 k a_{0} b_{1}^{3}+6 k a_{1} b_{0} b_{1}^{2}=0, \\
& \omega^{4} a_{0} \alpha_{3} b_{0}^{4}-4 \omega^{3} a_{0} \alpha_{2} b_{0}^{4}-12 \omega^{2} a_{0} \alpha_{1} b_{0}^{4}+24 \mu a_{0} b_{0}^{4}-24 a_{0}^{5} \beta_{2}-24 a_{0}^{3} b_{0}^{2} \beta_{1}=0, \\
& -(\ln (a))^{4} c^{4} a_{0} \alpha_{3} b_{0}^{3} b_{1}+(\ln (a))^{4} c^{4} a_{1} \alpha_{3} b_{0}^{4}+6(\ln (a))^{2} c^{2} \omega^{2} a_{0} \alpha_{3} b_{0}^{3} b_{1}-6(\ln (a))^{2} c^{2} \omega^{2} a_{1} \alpha_{3} b_{0}^{4} \\
& -12(\ln (a))^{2} c^{2} \omega a_{0} \alpha_{2} b_{0}^{3} b_{1}+12(\ln (a))^{2} c^{2} \omega a_{1} \alpha_{2} b_{0}^{4}-12(\ln (a))^{2} c^{2} a_{0} \alpha_{1} b_{0}^{3} b_{1}+12(\ln (a))^{2} c^{2} a_{1} \alpha_{1} b_{0}^{4} \\
& +4 \omega^{4} a_{0} \alpha_{3} b_{0}^{3} b_{1}+\omega^{4} a_{1} \alpha_{3} b_{0}^{4}-16 \omega^{3} a_{0} \alpha_{2} b_{0}^{3} b_{1}-4 \omega^{3} a_{1} \alpha_{2} b_{0}^{4}-48 \omega^{2} a_{0} \alpha_{1} b_{0}^{3} b_{1}-12 \omega^{2} a_{1} \alpha_{1} b_{0}^{4}+96 \mu a_{0} b_{0}^{3} b_{1} \\
& +24 \mu a_{1} b_{0}^{4}-120 a_{0}^{4} a_{1} \beta_{2}-48 a_{0}^{3} b_{0} b_{1} \beta_{1}-72 a_{0}^{2} a_{1} b_{0}^{2} \beta_{1}=0, \\
& 11(\ln (a))^{4} c^{4} a_{0} \alpha_{3} b_{0}^{2} b_{1}^{2}-11(\ln (a))^{4} c^{4} a_{1} \alpha_{3} b_{0}^{3} b_{1}+6(\ln (a))^{2} c^{2} \omega^{2} a_{0} \alpha_{3} b_{0}^{2} b_{1}^{2}-6(\ln (a))^{2} c^{2} \omega^{2} a_{1} \alpha_{3} b_{0}^{3} b_{1} \\
& -12(\ln (a))^{2} c^{2} \omega a_{0} \alpha_{2} b_{0}^{2} b_{1}^{2}+12(\ln (a))^{2} c^{2} \omega a_{1} \alpha_{2} b_{0}^{3} b_{1}-12(\ln (a))^{2} c^{2} a_{0} \alpha_{1} b_{0}^{2} b_{1}^{2}+12(\ln (a))^{2} c^{2} a_{1} \alpha_{1} b_{0}^{3} b_{1} \\
& +6 \omega^{4} a_{0} \alpha_{3} b_{0}^{2} b_{1}^{2}+4 \omega^{4} a_{1} \alpha_{3} b_{0}^{3} b_{1}-24 \omega^{3} a_{0} \alpha_{2} b_{0}^{2} b_{1}^{2}-16 \omega^{3} a_{1} \alpha_{2} b_{0}^{3} b_{1}-72 \omega^{2} a_{0} \alpha_{1} b_{0}^{2} b_{1}^{2}-48 \omega^{2} a_{1} \alpha_{1} b_{0}^{3} b_{1}+144 \mu a_{0} b_{0}^{2} b_{1}^{2} \\
& +96 \mu a_{1} b_{0}^{3} b_{1}-240 a_{0}^{3} a_{1}^{2} \beta_{2}-24 a_{0}^{3} b_{1}^{2} \beta_{1}-144 a_{0}^{2} a_{1} b_{0} b_{1} \beta_{1}-72 a_{0} a_{1}^{2} b_{0}^{2} \beta_{1}=0,
\end{aligned}
$$




$$
\begin{aligned}
& -11(\ln (a))^{4} c^{4} a_{0} \alpha_{3} b_{0} b_{1}^{3}+11(\ln (a))^{4} c^{4} a_{1} \alpha_{3} b_{0}^{2} b_{1}^{2}-6(\ln (a))^{2} c^{2} \omega^{2} a_{0} \alpha_{3} b_{0} b_{1}^{3}+6(\ln (a))^{2} c^{2} \omega^{2} a_{1} \alpha_{3} b_{0}^{2} b_{1}^{2} \\
& \quad+12(\ln (a))^{2} c^{2} \omega a_{0} \alpha_{2} b_{0} b_{1}^{3}-12(\ln (a))^{2} c^{2} \omega a_{1} \alpha_{2} b_{0}^{2} b_{1}^{2}+12(\ln (a))^{2} c^{2} a_{0} \alpha_{1} b_{0} b_{1}^{3}-12(\ln (a))^{2} c^{2} a_{1} \alpha_{1} b_{0}^{2} b_{1}^{2} \\
& \quad+4 \omega^{4} a_{0} \alpha_{3} b_{0} b_{1}^{3}+6 \omega^{4} a_{1} \alpha_{3} b_{0}^{2} b_{1}^{2}-16 \omega^{3} a_{0} \alpha_{2} b_{0} b_{1}^{3}-24 \omega^{3} a_{1} \alpha_{2} b_{0}^{2} b_{1}^{2}-48 \omega^{2} a_{0} \alpha_{1} b_{0} b_{1}^{3}-72 \omega^{2} a_{1} \alpha_{1} b_{0}^{2} b_{1}^{2}+96 \mu a_{0} b_{0} b_{1}^{3} \\
& \quad+144 \mu a_{1} b_{0}^{2} b_{1}^{2}-240 a_{0}^{2} a_{1}^{3} \beta_{2}-72 a_{0}^{2} a_{1} b_{1}^{2} \beta_{1}-144 a_{0} a_{1}^{2} b_{0} b_{1} \beta_{1}-24 a_{1}^{3} b_{0}^{2} \beta_{1}=0, \\
& (\ln (a))^{4} c^{4} a_{0} \alpha_{3} b_{1}^{4}-(\ln (a))^{4} c^{4} a_{1} \alpha_{3} b_{0} b_{1}^{3}-6(\ln (a))^{2} c^{2} \omega^{2} a_{0} \alpha_{3} b_{1}^{4}+6(\ln (a))^{2} c^{2} \omega^{2} a_{1} \alpha_{3} b_{0} b_{1}^{3}+12(\ln (a))^{2} c^{2} \omega a_{0} \alpha_{2} b_{1}^{4} \\
& \quad-12(\ln (a))^{2} c^{2} \omega a_{1} \alpha_{2} b_{0} b_{1}^{3}+12(\ln (a))^{2} c^{2} a_{0} \alpha_{1} b_{1}^{4}-12(\ln (a))^{2} c^{2} a_{1} \alpha_{1} b_{0} b_{1}^{3}+\omega^{4} a_{0} \alpha_{3} b_{1}^{4}+4 \omega^{4} a_{1} \alpha_{3} b_{0} b_{1}^{3} \\
& \quad-4 \omega^{3} a_{0} \alpha_{2} b_{1}^{4}-16 \omega^{3} a_{1} \alpha_{2} b_{0} b_{1}^{3}-12 \omega^{2} a_{0} \alpha_{1} b_{1}^{4}-48 \omega^{2} a_{1} \alpha_{1} b_{0} b_{1}^{3}+24 \mu a_{0} b_{1}^{4}+96 \mu a_{1} b_{0} b_{1}^{3}-120 a_{0} a_{1}^{4} \beta_{2} \\
& \quad-72 a_{0} a_{1}^{2} b_{1}^{2} \beta_{1}-48 a_{1}^{3} b_{0} b_{1} \beta_{1}=0, \\
& \omega^{4} a_{1} \alpha_{3} b_{1}^{4}-4 \omega^{3} a_{1} \alpha_{2} b_{1}^{4}-12 \omega^{2} a_{1} \alpha_{1} b_{1}^{4}+24 \mu a_{1} b_{1}^{4}-24 a_{1}^{5} \beta_{2}-24 a_{1}^{3} b_{1}^{2} \beta_{1}=0,
\end{aligned}
$$

whose solution yields the following exact solutions:

\section{Case 1}

$u_{1,2}(z, t)=\frac{a_{0}+a_{1} a^{k z-c t}}{b_{0}+b_{1} a^{k z-c t}} \mathrm{e}^{\mathrm{i}(\mu z-\omega t)}$,

in which

$$
\begin{aligned}
& a_{0}=-\frac{\sqrt{30} b_{0}}{10 \alpha_{3} \beta_{2}} \sqrt{\beta_{2}\left(-2 \beta_{1} \alpha_{3}^{2}+\sigma\right)}, \\
& a_{1}=\frac{\sqrt{30} b_{1}}{10 \alpha_{3} \beta_{2}} \sqrt{\beta_{2}\left(-2 \beta_{1} \alpha_{3}^{2}+\sigma\right)}, \\
& k= \pm \frac{\alpha_{2}\left(3 \alpha_{1} \alpha_{3}+\alpha_{2}^{2}\right)}{3 \alpha_{3}^{3} \ln (a)} \\
& \times \sqrt{-\frac{-24 \alpha_{1}^{2} \alpha_{3}^{2} \beta_{2}-24 \alpha_{1} \alpha_{2}^{2} \alpha_{3} \beta_{2}-6 \alpha_{2}^{4} \beta_{2}+12 \sigma \alpha_{3} \beta_{1}}{10 \alpha_{1} \alpha_{3} \beta_{2}+5 \alpha_{2}^{2} \beta_{2}}}, \\
& c= \pm \frac{1}{\alpha_{3} \ln (a)} \\
& \times \sqrt{-\frac{-24 \alpha_{1}^{2} \alpha_{3}^{2} \beta_{2}-24 \alpha_{1} \alpha_{2}^{2} \alpha_{3} \beta_{2}-6 \alpha_{2}^{4} \beta_{2}+12 \sigma \alpha_{3} \beta_{1}}{10 \alpha_{1} \alpha_{3} \beta_{2}+5 \alpha_{2}^{2} \beta_{2}}}, \\
& \mu=-\left(12 \alpha_{3}\left(-2 \beta_{1} \alpha_{3}^{2}+\sigma\right) \beta_{1}-72 \alpha_{1}^{2} \alpha_{3}^{2} \beta_{2}\right. \\
& \left.-172 \alpha_{1} \alpha_{2}^{2} \alpha_{3} \beta_{2}-43 \alpha_{2}^{4} \beta_{2}+72 \alpha_{3}^{3} \beta_{1}^{2}\right)\left(200 \beta_{2} \alpha_{3}^{3}\right)^{-1}, \\
& \omega=\frac{\alpha_{2}}{\alpha_{3}}, \\
& \sigma=\sqrt{4 \alpha_{1}^{2} \alpha_{3}^{3} \beta_{2}+4 \alpha_{1} \alpha_{2}^{2} \alpha_{3}^{2} \beta_{2}+\alpha_{2}^{4} \alpha_{3} \beta_{2}},
\end{aligned}
$$

\section{Case 2}

$u_{3,4}(z, t)=\frac{a_{0}+a_{1} a^{k z-c t}}{b_{0}+b_{1} a^{k z-c t}} \mathrm{e}^{\mathrm{i}(\mu z-\omega t)}$, in which

$$
\begin{aligned}
& a_{0}=\frac{\sqrt{30} b_{0}}{10 \alpha_{3} \beta_{2}} \sqrt{\beta_{2}\left(-2 \beta_{1} \alpha_{3}^{2}+\sigma\right)}, \\
& a_{1}=-\frac{\sqrt{30} b_{1}}{10 \alpha_{3} \beta_{2}} \sqrt{\beta_{2}\left(-2 \beta_{1} \alpha_{3}^{2}+\sigma\right)}, \\
& k= \pm \frac{\alpha_{2}\left(3 \alpha_{1} \alpha_{3}+\alpha_{2}^{2}\right)}{3 \alpha_{3}^{3} \ln (a)} \\
& \times \sqrt{-\frac{-24 \alpha_{1}^{2} \alpha_{3}^{2} \beta_{2}-24 \alpha_{1} \alpha_{2}^{2} \alpha_{3} \beta_{2}-6 \alpha_{2}^{4} \beta_{2}+12 \sigma \alpha_{3} \beta_{1}}{10 \alpha_{1} \alpha_{3} \beta_{2}+5 \alpha_{2}^{2} \beta_{2}}}, \\
& c= \pm \frac{1}{\alpha_{3} \ln (a)} \\
& \times \sqrt{-\frac{-24 \alpha_{1}^{2} \alpha_{3}^{2} \beta_{2}-24 \alpha_{1} \alpha_{2}^{2} \alpha_{3} \beta_{2}-6 \alpha_{2}^{4} \beta_{2}+12 \sigma \alpha_{3} \beta_{1}}{10 \alpha_{1} \alpha_{3} \beta_{2}+5 \alpha_{2}^{2} \beta_{2}}}, \\
& \mu=-\left(12 \alpha_{3}\left(-2 \beta_{1} \alpha_{3}^{2}+\sigma\right) \beta_{1}-72 \alpha_{1}^{2} \alpha_{3}^{2} \beta_{2}-172 \alpha_{1} \alpha_{2}^{2} \alpha_{3} \beta_{2}\right. \\
& \left.-43 \alpha_{2}^{4} \beta_{2}+72 \alpha_{3}^{3} \beta_{1}^{2}\right)\left(200 \beta_{2} \alpha_{3}^{3}\right)^{-1}, \\
& \omega=\frac{\alpha_{2}}{\alpha_{3}}, \\
& \sigma=\sqrt{4 \alpha_{1}^{2} \alpha_{3}^{3} \beta_{2}+4 \alpha_{1} \alpha_{2}^{2} \alpha_{3}^{2} \beta_{2}+\alpha_{2}^{4} \alpha_{3} \beta_{2}},
\end{aligned}
$$

\section{Numerical simulations and physical properties}

In this section, numerical simulations are presented to illustrate the physical properties of the obtained solutions. For instance, the solutions $u_{1}(z, t)$ and $u_{3}(z, t)$ have been considered, respectively, in Figs. 1 and 2 


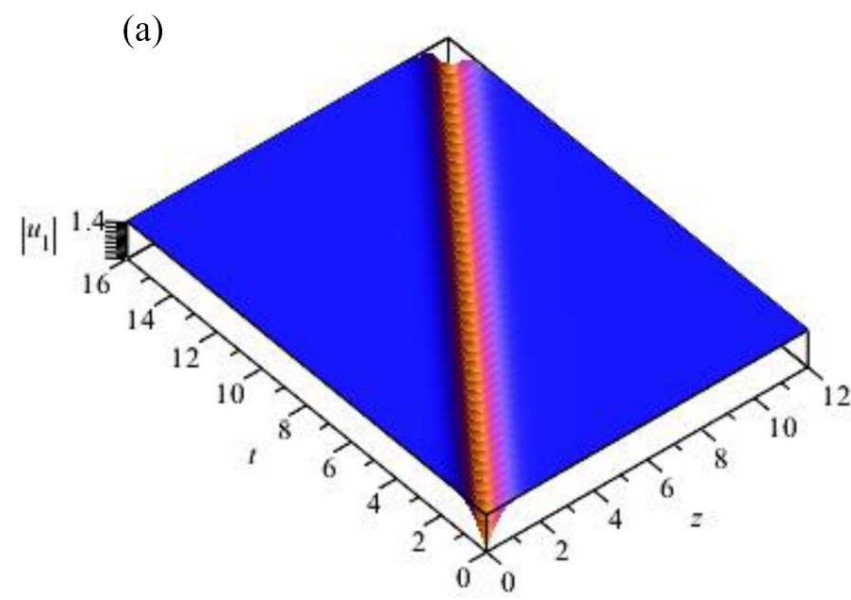

(b)

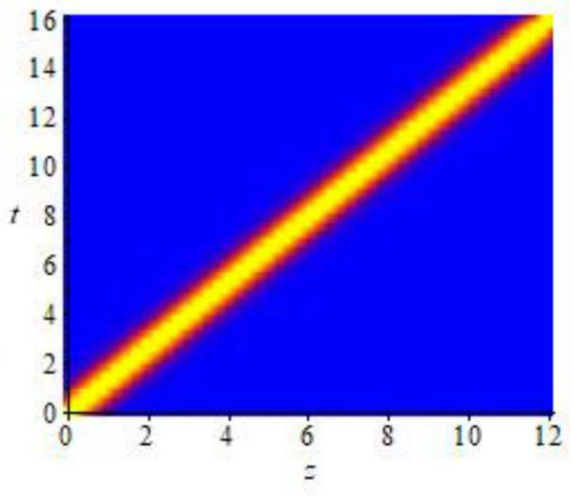

Fig. 1. The solution $u_{1}(z, t)$ when $b_{0}=b_{1}=\alpha_{1}=\alpha_{2}=\alpha_{3}=1, \beta_{1}=-2, \beta_{2}=1$, and $a=2$ : (a) 3D plot, (b) density plot.

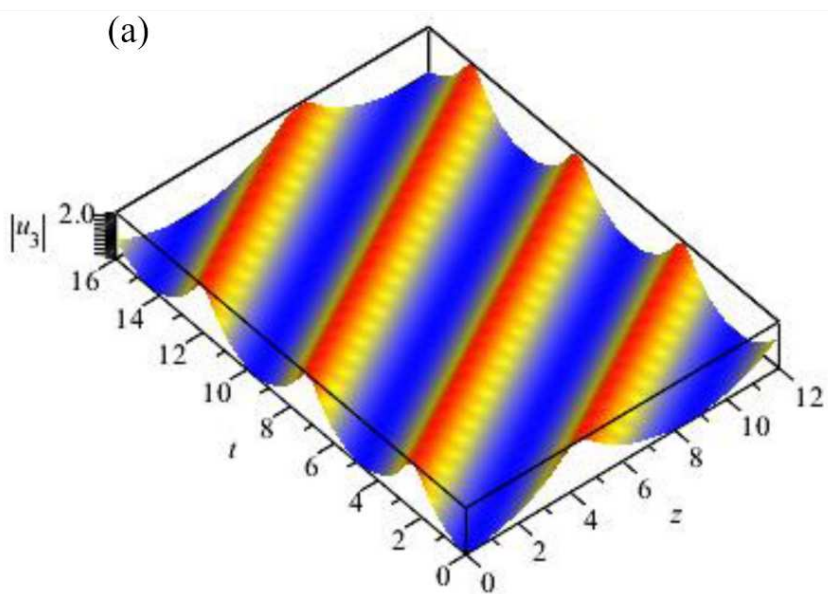

(b)

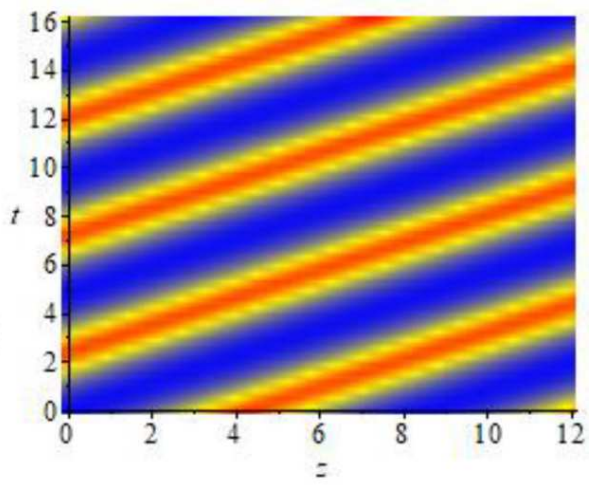

Fig. 2. The solution $u_{3}(z, t)$ when $b_{0}=1, b_{1}=2, \alpha_{1}=1.1, \alpha_{2}=0.7, \alpha_{3}=1.5, \beta_{1}=1.9, \beta_{2}=1$, and $a=2$ : (a) 3D plot, (b) density plot.

for some opted parameters. Figure 1 shows a dark solitary wave solution, whereas Fig. 2 evinces a periodic solitary wave solution. In the normal dispersion region, solitons are transmitted as dark ones, and conversely, in the anomalous dispersion region, solitons are transmitted as bright ones [28]. Dark solitons are chirped, viz., the phase of a dark soliton varies across its width, whereas bright solitons have a constant phase [29]. Moreover, the velocity of a dark soliton relates to its amplitude through the internal phase angle [29]. Both dark and bright solitons in a single equation model result from the self phase modulation.

\section{Conclusion}

The light pulse propagation in optical fibers was studied in the present paper. In this regard, the nonlinear governing model which is known as the dispersive cubic-quintic nonlinear Schrödinger equation including high-order time derivatives was solved successfully. The $\exp _{a}$-function scheme along with a symbolic computation system was formally employed to extract the exact solutions of the model. As an upshot, a wide range of exact solutions including dark and periodic solitary wave solutions were derived, proving the excellent performance of the scheme.

\section{References}

[1] N.A. Kudryashov, Commun. Nonlin. Sci. Numer. Simulat. 17, 2248 (2012).

[2] M. Mirzazadeh, M. Eslami, A. Biswas, Optik 125, 6874 (2014).

[3] M. Mirzazadeh, R.T. Alqahtani, A. Biswas, Optik 145, 74 (2017).

[4] K. Hosseini, P. Mayeli, D. Kumar, J. Mod. Opt. 65, 361 (2018).

[5] K. Hosseini, F. Samadani, D. Kumar, M. Faridi, Optik 157, 1101 (2018). 
[6] A. Biswas, A. Sonmezoglu, M. Ekici, M. Mirzazadeh, Q. Zhou, S.P. Moshokoa, M. Belic, Optik 164, 303 (2018).

[7] M. Kaplan, K. Hosseini, F. Samadani, N. Raza J. Mod. Opt. 65, 1431 (2018).

[8] A. Bekir, A.C. Cevikel, Commun. Nonlin. Sci. Numer. Simulat. 14, 1804 (2009).

[9] A.M. Wazwaz, Appl. Math. Computat. 200, 160 (2008).

[10] A. Biswas, Y. Yildrium, E. Yaser, Q. Zhou, S.P. Moshokoa, M. Belic, Optik 160, 24 (2018).

[11] A. Biswas, Y. Yildrium, E. Yaser, H. Triki, A.S. Alshomrani, M.Z. Ullah, Q. Zhou, S.P. Moshokoa, M. Belic, Optik 157, 1235 (2018).

[12] S. Bibi, S.T. Mohyud-Din, J. Assoc. Arab Univ. Basic Appl. Sci. 15, 90 (2014).

[13] K.R. Raslan, T.E. El-Danaf, K.K. Ali, J. Egypt. Math. Soc. 25, 350 (2017).

[14] W.X. Ma, J.H. Lee, Chaos Solitons Fractals 42, 1356 (2009).

[15] S. Zhang, H.Q. Zhang, Pramana J. Phys. 76, 561 (2011).

[16] O. Guner, Optik 130, 448 (2017).

[17] K. Hosseini, P. Mayeli, R. Ansari, Waves Random Complex Media 28, 426 (2018).
[18] A. Akbulut, M. Kaplan, Comput. Math. Appl. 75, 876 (2018).

[19] E.M.E. Zayed, K.A.E. Alurrfi, Optik 127, 9131 (2016).

[20] R. Kohl, D. Milovic, E. Zerrad, A. Biswas, J. Infrared Millim. Terahertz Waves 30, 526 (2009).

[21] A. Biswas, M.Z. Ullah, M. Asma, Q. Zhou, S.P. Moshokoa, M. Belic, Optik 139, 16 (2017).

[22] A.T. Ali, E.R. Hassan, Appl. Math. Computat. 217, $451(2010)$.

[23] K. Hosseini, A. Zabihi, F. Samadani, R. Ansari, Opt. Quant. Electron. 50, 82 (2018)

[24] K. Hosseini, Z. Ayati, R. Ansari, J. Mod. Opt. 65 , 847 (2018).

[25] C.Q. Dai, J.L. Chen, J.F. Zhang, Int. J. Mod. Phys. B 21, 2657 (2007)

[26] F. Azzouzi, H. Triki, K. Mezghiche, A. El Akrmi, Chaos Solitons Fractals 39, 1304 (2009).

[27] Y. Xie, Z. Yang, L. Li, Phys. Lett. A 382, 2506 (2018).

[28] W. Yu, M. Ekici, M. Mirzazadeh, Q. Zhou, W. Liu, Optik 165, 341 (2018).

[29] P.L. Christiansen, M.P. Sørensen, A.C. Scott, Nonlinear Science at the Dawn of the 21st Century, Springer, 2000 